\title{
Usual and Unusual Effects in ADF-STEM Imaging of Dopant Atom in Crystals
}

\author{
Anudha Mittal, Aloysius A. Gunawan, and K. Andre Mkhoyan
}

Department of Chemical Engineering and Materials Science, University of Minnesota, Minneapolis, MN 55455.

Interest in imaging and identifying individual dopant atoms inside crystalline specimens has a long history in electron microscopy. While both conventional transmission electron microscopes (TEMs) and scanning transmission electron microscopes (STEMs) are capable of imaging an individual atom [1], detecting an individual dopant atom inside the crystal appears to be easiest using an annular dark field detector in STEM. With recent advances in lens aberration correction ADF-STEM imaging has become applicable even for crystalline sample with $<0.1 \mathrm{~nm}$ spacing between atomic columns.

Imaging dopant atoms in crystalline specimens is complicated by sample-sensitive changes in the incident electron beam due to channelling [2]. However, despite these challenges, several groups have successfully imaged dopant atoms inside a host: Voyles et al. [3] observed $\mathrm{Sb}$ atoms inside Si, Varela et al. [4] studied La-doped $\mathrm{CaTiO}_{3}$, Shibata et al. [5] imaged $\mathrm{Y}$ atoms in $\mathrm{Al}_{2} \mathrm{O}_{3}$, and Lupini et al. [6] studied Bi dopant atoms in Si. Yet, the number of cases reported in literature is too few to develop a systematic view on parameters and conditions that govern visibility of individual dopant atom in ADF-STEM images. Understanding the roles of the microscope parameters and specimen conditions are instrumental in designing experiments to detect individual dopant atoms and determine their location as precisely as possible to reconstruct the atomic structure of the doped material.

We have investigated the limits of ADF-STEM imaging to determine presence and position of individual dopant atoms inside the host crystal by analysing simulated using the multislice method ADFSTEM images. Specimen features, such as thickness and crystallographic orientation of the host material, position of a dopant atom inside a specimen, Z-difference between dopant element and host, and probe parameters that affect the visibility of a dopant atom have been examined. This analysis provides a guide for optimization of the conditions for improving detection of a dopant atom and assessment of the conditions under which a dopant atom is not detectable at all in ADF-STEM images.

The relationship between the position of a dopant atom and its visibility in ADF-STEM image is not simple: several different locations of dopant atom can lead to the same contrast between doped and non-doped atomic columns. For instance, an Sn dopant atom located on the entrance surface in $3 \mathrm{~nm}$ thick Si [110] has $66 \%$ visibility. Sn dopant atom, located 2.7 below the entrance surface, in $5 \mathrm{~nm}$ thick Si [110] has also 66\% visibility. Moreover, different types of dopant atoms can also have the same calculated visibility. For example, a Ge dopant atom, located $1.2 \mathrm{~nm}$ below the entrance surface in a $2 \mathrm{~nm}$ thick Si oriented long [110] direction also has $66 \%$ visibility. Thus, determination of dopant atom position requires at least knowledge of dopant identity and specimen thickness. However, even with specimen thickness, dopant element, and probe conditions known, two different dopant atom positions can still lead to the same visibility (see Fig. 1).

Another result showing the extent to which intensity of doped columns can be counter-intuitive is the presence of negative values of visibility at certain specimen thicknesses although $Z_{\text {Dopant }}>Z_{\text {Host. }}$. For instance, Sn dopant atom at $9 \mathrm{~nm}$ depth in $25 \mathrm{~nm}$ thick Si [111] has $-4 \backslash \%$ visibility (see Fig. 2). Comparison of beam intensity profiles of doped and pure columns shows that the beam intensity of a doped column is less than the intensity of a pure column at certain specimen thicknesses, as shown in Fig. 3. Atoms exposed to fewer incident electrons will contribute less to ADF image intensity. Hence, a doped column can have lower intensity than the pure host column even though $Z_{\text {Dopant }}>Z_{\text {Host. }}$ Similarly, a doped column can have higher intensity than a pure column when $Z_{\text {Dopant }}<Z_{\text {Host }}$ (see Fig. 4) [7].

\section{References}

[1] A.V. Crewe, J. Wall, and J. Langmore, Science 168 (1970) 1338.

[2] J. Fertig and H. Rose, Optik 59 (1981) 407. 
[3] P.M. Voyles et al., Nature 416 (2002) 826.

[4] M. Varela et al., Phys. Rev. Lett. 92 (2004) 095502.

[5] N. Shibata et al., Nat. Mater. 8 (2009) 654.

[6] A.R. Lupini et al., Microsc. Microanal. 15 (2009) 441.

[7] This work was supported partially by the NSF-MRSEC DMR-0819885. We acknowledge receiving technical help from Dr. Ravi Chityala and access to computational resources from University of Minnesota Supercomputing Institute.
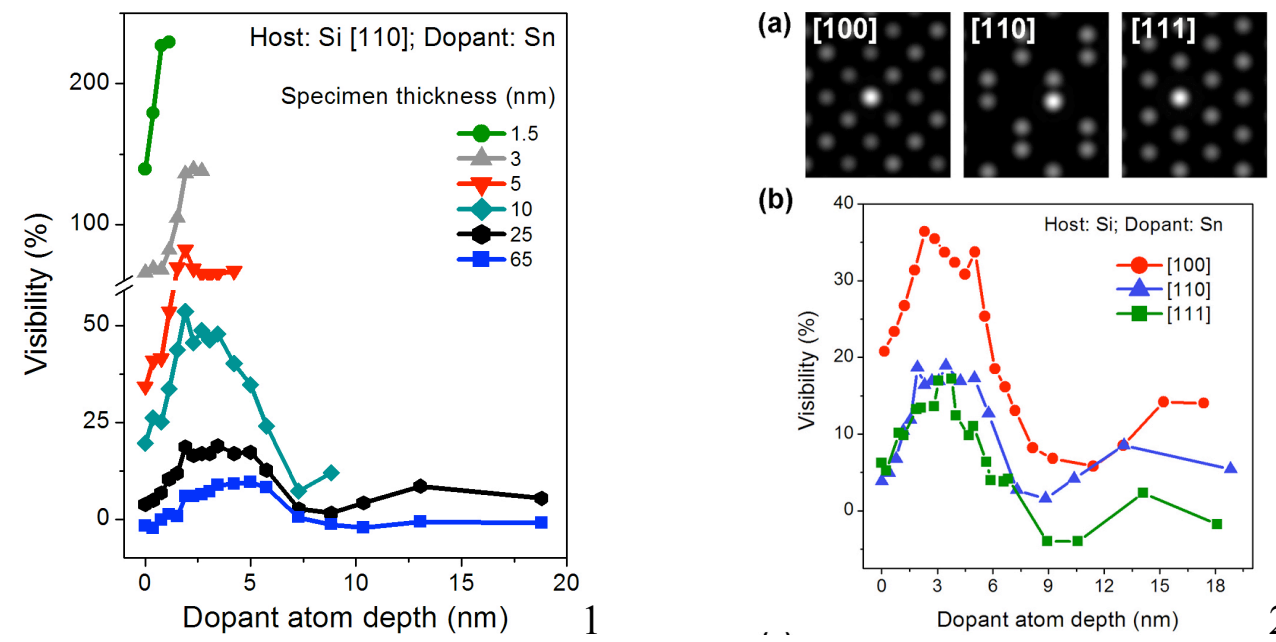

(b)
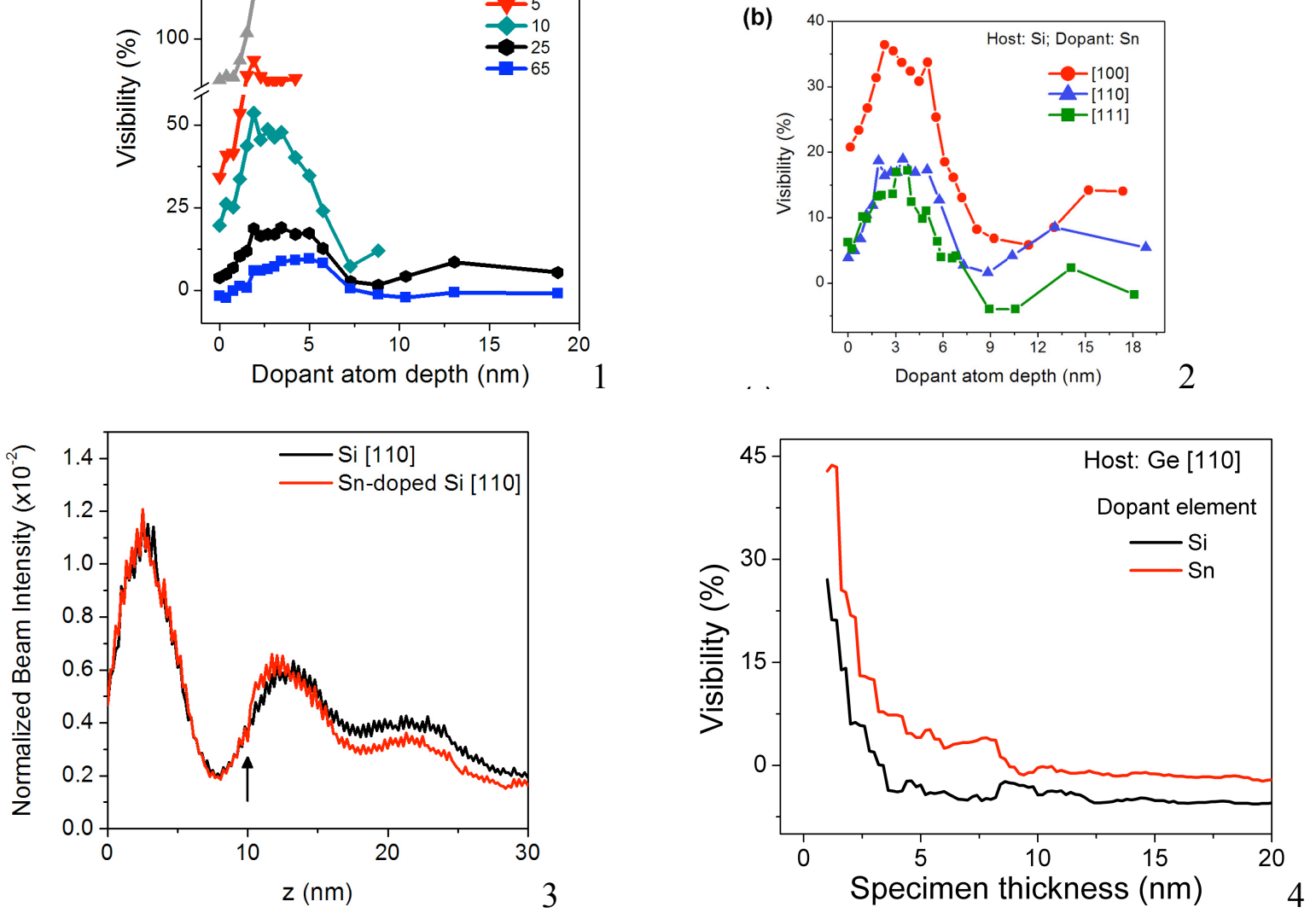

Fig. 1. Dopant atom visibility in ADF-STEM images as a function of dopant atom depth for Sn-doped Si viewed in [110] orientation.

Fig. 2. (a) ADF-STEM images of $3 \mathrm{~nm}$ thick Si specimens with single $\mathrm{Sn}$ dopant atom located $2.7 \mathrm{~nm}$ below the entrance surface viewed along [100], [110] and [111] low order zone-axes. (b) Visibility of Sn atom in ADF-STEM images as a function of dopant atom depth for these three crystallographic orientations.

Fig. 3. Incident beam intensity as electrons of the probe, located on the atomic column, propagate along [110] crystallographic orientations in Si host with and without Sn dopant atom. Position of the dopant atom is indicated with an arrow $(\mathrm{d}=10 \mathrm{~nm})$.

Fig. 4. Visibility of dopant Si and Sn dopant atoms in ADF-STEM images located $0.8 \mathrm{~nm}$ from the entrance surface in $25 \mathrm{~nm}$ thick Ge [110] as a function of specimen thickness. 